Revue française de la traduction

Le vernaculaire chiac de Moncton en traduction littéraire : l'exemple de Petites difficultés d'existence de France Daigle

\title{
Henri Biahé
}

\section{(2) OpenEdition \\ Journals}

\section{Édition électronique}

URL : http://journals.openedition.org/traduire/90

DOI : $10.4000 /$ traduire.90

ISSN : 2272-9992

Éditeur

Société française des traducteurs

\section{Édition imprimée}

Date de publication : 15 décembre 2011

Pagination : 66-79

ISBN : 039-773X

ISSN : 0395-773X

Référence électronique

Henri Biahé, «Le vernaculaire chiac de Moncton en traduction littéraire : l'exemple de Petites difficultés d'existence de France Daigle », Traduire [En ligne], 225 | 2011, mis en ligne le 10 février 2014, consulté le 20 avril 2019. URL : http://journals.openedition.org/traduire/90 ; DOI : 10.4000/traduire.90 


\section{Le vernaculaire chiac de Moncton en traduction littéraire : l'exemple de Petites difficultés d'existence de France Daigle}

Henri Biahé

\section{Introduction}

La traduction de textes linguistiquement hybrides produits par les écrivains dominés ou des "littératures de l'exiguité " (Paré : 1992) n'est pas chose aisée. Comment Robert Majzels parvient-il à traduire avec bonheur le vernaculaire chiac - parlé au sud-est du Nouveau-Brunswick au Canada - présent dans le roman de France Daigle intitulé Petites difficultés d'existence, publié en 2002 et traduit en anglais deux années plus tard sous le titre Life's Little Difficulties ? Ce travail entend d'abord dresser la situation sociolinguistique de l'environnement dans lequel évolue le chiac, puis procéder à une brève analyse des caractéristiques linguistiques de ce parler. Seront également abordées la problématique de la traduction de ce vernaculaire et les stratégies utilisées pour mener à bien la traduction. Enfin, on procédera à l'esquisse d'une approche de la traduction spécifiquement applicable au chiac.

\section{Portrait sociolinguistique}

S'il est vrai qu'à ce jour l'origine du terme " chiac " reste assez floue, l'on sait en revanche que ce nom renvoie à un vernaculaire hybride à base de français, ayant recours à des formes lexicales, syntaxiques, morphologiques et phoniques de l'anglais, et parlé au sud-est du NouveauBrunswick, l'unique province canadienne officiellement bilingue. On le voit, le chiac est une langue en contact avec les deux langues indoeuropéennes susévoquées, dans une région où les francophones sont statistiquement minoritaires (33\%) par rapport à l'écrasante majorité anglophone. En effet, le chiac naît de la conjonction d'un certain nombre de facteurs assez récents constatés à Moncton - principale métropole de la province - qui ont favorisé le développement de cette variété autonome du français. Perrot (2001: 130) le souligne opportunément en ces termes:

Au plan linguistique, Moncton se caractérise par un continuum complexe qui est le produit de récentes évolutions : 1) l'affaiblissement de l'acadien traditionnel, encore parlé par la plus vieille 
génération (certains traits "archaïques" restent cependant très vivaces, même chez les jeunes); 2) le développement d'un français régional plus normatif, langue du système éducatif et des médias ; 3) l'intensification du contact avec l'anglais et la pratique du bilinguisme en milieu urbain minoritaire. Ce contexte a donné naissance à une variété mixte distincte communément appelée chiac et souvent décrite par les locuteurs eux-mêmes comme "les deux langues mêlées ".

Dans la réalité, le chiac qui s'appuie sur une "matrice française " (Perrot : 2001) est surtout utilisé par de jeunes francophones bilingues anglais-français (Young 2002 ; Pöll 2009). Pas étonnant que ce code mixte relativement stabilisé soit identifié par ses locuteurs comme une langue qui véhicule une identité francophone. D'ailleurs, Perrot $(2005: 318)$ relève que " dans les représentations des locuteurs, le chiac est généralement perçu comme une variété de français véhiculant une identité francophone particulière (le "nouveau français de Moncton", selon l'un des informateurs du corpus 2001)".

Certes, pour une majorité de locuteurs, le chiac véhicule une identité francophone. II existe cependant un discours antichiac qui ne voit pas d'un bon œil le mélange de langues caractéristique de ce parler. À ce sujet, Boudreau (2008 : 64) affirme : "Dans l'esprit de bon nombre

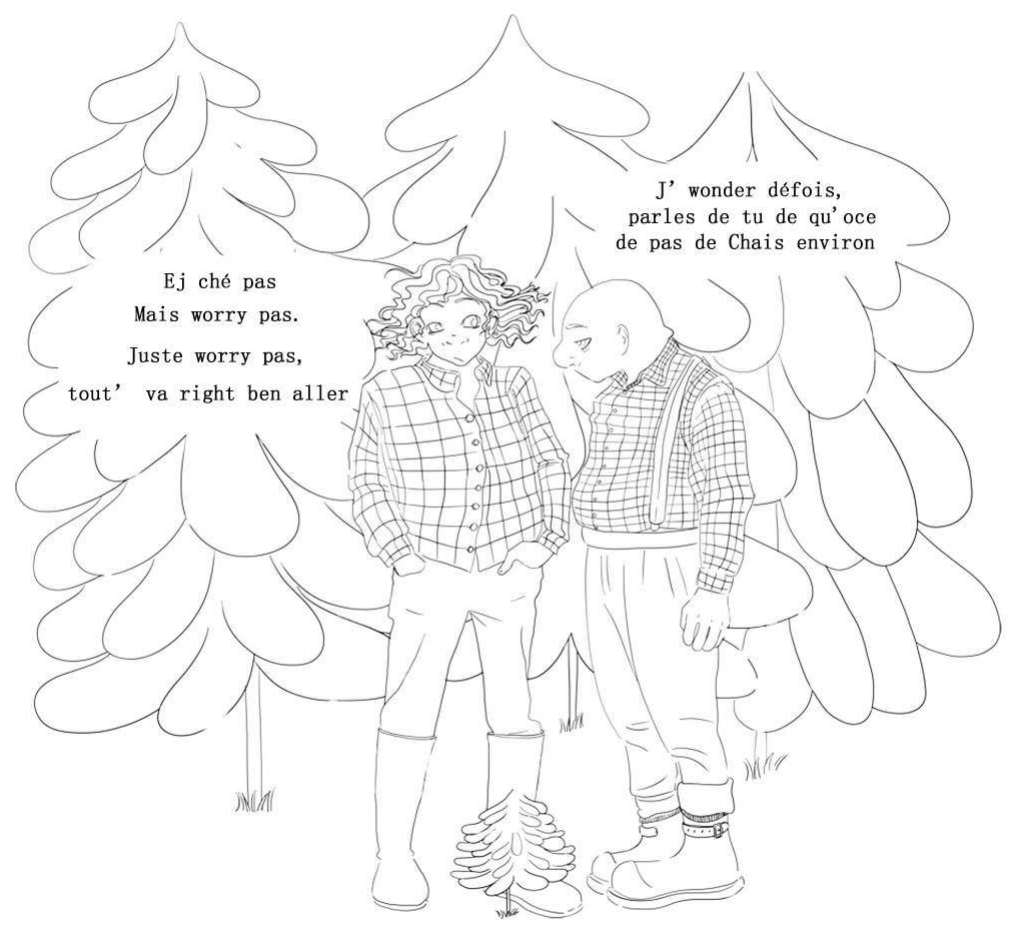

Illustration : Marlène Junius, http://alotoftralala.over-blog.com 
de personnes, il [le chiac] symbolise l'assimilation et l'acculturation et nourrit les discours traditionnels voulant que l'impureté et le mélange mènent à la perte de repères linguistiques et culturels. " Relayant les commentaires épilinguistiques d'autres personnes critiques à l'égard de ce code mixte, Boudreau et Gadet (1998) font remarquer que le chiac est souvent perçu comme " un ghetto linguistique qui risque d'isoler les Acadiens des autres francophones". En dépit de la stigmatisation dont ce sociolecte est l'objet, il n'en demeure pas moins que ces dernières années, l'on observe des actions qui tendent à le légitimer, comme le souligne justement Perrot (2005: 321) : " Je l'ai dit, même si sa reconnaissance n'est encore que partielle, le chiac n'est plus le tabou linguistique qu'il a été et il s'est fait une place, ces dernières années, dans le paysage linguistique monctonien. " Au rang des principaux acteurs qui travaillent à la légitimation du vernaculaire du sud-est du Nouveau-Brunswick figurent les artistes, les promoteurs culturels, les poètes et romanciers, etc. D'après Leblanc (2003: 520) cité par Leclerc (2008: 164), cette entreprise de légitimation vise à " transform[er] cet objet de mépris en une force d'expression qui témoigne d'une réalité vécue, articulée et assumée ". Leclerc (ibid.) ajoute que " [s]ur la scène littéraire, l'une des conséquences les plus remarquables de ce travail de légitimation est l'emploi de plus en plus fréquent du chiac, langue d'abord orale et populaire, dans des textes romanesques ". Avant de se pencher sur l'emploi du chiac dans l'univers romanesque et sa traduction en anglais, objet du présent article, il ne serait pas superflu de procéder rapidement à une description linguistique de ce parler.

\section{Quelques caractéristiques linguistiques}

La description linguistique qui suit est loin d'être exhaustive. Toutefois, elle permet de souligner à grands traits quelques caractéristiques qui attestent du degré de stabilisation du chiac. Certaines définitions pour le moins approximatives des formes linguistiques du vernaculaire du sud-est du Nouveau-Brunswick considèrent ce parler comme un mélange hétéroclite d'anglais et de français parlés "randomly throughout " (Ball, 2004, cité par Malaborza, 2006 : 199), ce qui pourrait faire penser que le chiac se réduirait à une simple alternance de codes. Pourtant, Perrot (1995, 1998, 2005), qui reste à ce jour l'une des rares spécialistes du chiac à avoir procédé à une analyse linguistique exhaustive de ce code mixte, a pu démontrer avec force détails qu'il existe des productions orales de locuteurs du chiac qui peuvent être effectivement considérées comme des phénomènes d'alternance codique intra et interphrastique. Les exemples ci-après en témoignent :

"Yeah moi j'étais de même aussi / j'avais des longs cheveux / jeez you're burnt man " (Perrot, $319: 2005)$.

"Yeah des preps veniont à moi des fois hein / pi i disiont heu / that's 'cause you got long hair you have to wear your stupid ripped up jeans / your jean jacket there with the patches on it. " (Perrot, 319 : 2005). 
Cependant, l'auteure fait remarquer que les cas d'alternance codique sont essentiellement marginaux et imprévisibles. En appui à sa thèse, elle fait siens les commentaires épilinguistiques de locuteurs du chiac qui établissent eux-mêmes une différence nette entre ce qu'ils considèrent d'une part comme du "pas tout à fait " ou " pas vraiment " chiac, qui se caractérise essentiellement par une juxtaposition de l'anglais et du français, et d'autre part comme du " vrai chiac " ou " chiac correct ", marqué par des formes linguistiques récurrentes. À propos de ces formes linguistiques remarquablement stabilisées, nous nous attarderons un tant soit peu sur quelques connecteurs et ponctuants, sur des lexèmes verbaux du nom ainsi que sur l'adjectif.

\section{a. Connecteurs et ponctuants du discours}

II s'agit essentiellement des connecteurs but (mais) et well (eh bien), anyway (en tout cas), etc., qui, selon Perrot (314 : 2005), sont des emprunts totalement stabilisés et participent du même coup à l'élaboration de la structure discursive et argumentative du chiac, comme le montrent les exemples ci-dessous :

"Y dort. Je l'amené au café tantôt... Je sais pas si c'est à cause du last storm ou pas, but le monde était grouchy là. " (Daigle, 142 : 2002).

"Me semblait aussi qu'à l'était pâle hier ! Awh well, on s'arrangera. Occupe-toi ben de yelle. A m'a sauvé la vie, c'te femme-là. " (Daigle, 91 : 2002).

"So, c'a tout' commencé avec c'te guy-icitte _ un paromaniac. Qu'a brûlé la maison d'un artiste. " (Daigle, 142 : 2002).

" J'aide juste Zed. Je suis en chômage, j'ai pas grand-chose d'autre à faire. Pis anyway, j'y ai dit que t'étais pas folle folle de l'idée. " (Daigle, 88 : 2002).

\section{b. Lexèmes verbaux}

Perrot (ibid. : 314) distingue les lexèmes dits simples (watch, move, drive, clean) et les lexèmes verbaux composés qui sont en général des verbes suivis d'une particule adverbiale (help out, help around). Reprenant à son propre compte Peronnet (1989), l'auteure confirme le constat selon lequel le verbe anglais est systématiquement adapté à la conjugaison franco-acadienne des verbes considérés comme faisant partie du premier groupe :

"Ça me tente de clean-er. "

" Je le tap-ais, une show qu'à start-é, je l'ai watch-é. " (Perrot, 314 : 2005).

S'agissant des verbes à particules, la particule adverbiale anglaise back ajoutée à un verbe français permet d'obtenir des structures propres au chiac, qu'on ne retrouverait ni en français ni en anglais :

"C'est-tu encore on ça ?" (Est-ce que ça passe encore ?)

"Non but c'est back suppose venir on. " (C'est censé repasser) (Perrot, 315 : 2005). 
On le voit, l'adverbe back qui précède le verbe français dans l'exemple ci-dessus a un sens itératif en chiac. Par contre, en anglais, il indique normalement le retour à un lieu ou un état antérieur. En français, la valeur itérative s'exprime généralement au moyen d'un préfixe verbal (par exemple : recommencer, redémarrer).

\section{c. Substantifs et adjectifs}

En chiac, le substantif anglais est généralement précédé d'un déterminant français. Ce dernier indique toujours la marque du nombre ou du genre du nom en question. Par ailleurs, il existe une majorité de locuteurs chiac qui tend à prononcer le " $\mathrm{S}$ " du pluriel des noms anglais.

"Ma day est jam-packed. " (Young, 109 : 2002).

"Ma gang peut s'accorder avec anybody / comme mes friendS personally. " (Perrot, 315 : 2005).

L'adjectif anglais reste invariable et précède le nom, quelle que soit l'origine de ce dernier :

"Mes normal shows, [j'ai pas de] favorite programme. "(Perrot, 316 : 2005).

\section{Une traduction du chiac est-elle possible ?}

Le chiac a rarement été abordé dans une perspective traductologique, hormis une poignée d'articles. Pourtant, ce parler pose un certain nombre de difficultés au traducteur, en raison de son statut de vernaculaire ou de sociolecte d'une part, et de l'hybridation linguistique qui le caractérise d'autre part. Dans le premier cas de figure, Berman (1985: 79) affirme : " Malheureusement, le vernaculaire, collant au terroir, résiste à toute traduction directe dans un autre vernaculaire. Seules les langues "langues cultivées" peuvent s'entretraduire. "Dans le second cas, Derrida (1985: 215) renchérit : " Comment traduire un texte écrit en plusieurs langues à la fois ? Comment "rendre" l'effet de pluralité ? Et si l'on traduit par plusieurs langues à la fois, appelle-t-on cela traduire ? " De ce qui précède, il ressort que les deux auteurs cités posent clairement le problème de l'intraduisibilité des textes linguistiquement hybrides et des vernaculaires. Dès lors, on est en droit de se poser la question de savoir si le chiac qui correspond au type de textes décrit par Derrida et Berman est effectivement intraduisible. Les parlers hybrides ou vernaculaires renvoient à une fonction référentielle dans la mesure où ils sont utilisés quotidiennement par leurs locuteurs, le plus souvent à des fins de communication. Cependant, l'inscription formelle de ces codes dans les œuvres littéraires par des auteurs issus des littératures mineures ou de "l'exiguitté ", comme dans le cas du roman étudié dans cet article, relève en général d'une entreprise délibérée des auteurs au sens où " to encounter language neutrally" (Batchelor, 2009 : 2) semble être un défi difficile à relever. Dans une telle situation, la présence de parlers hybrides dans une œuvre de fiction peut souvent revêtir plusieurs fonctions : identitaire, subversive, idéologique, esthétique, etc., lesquelles ne sont pas 
toujours aisément transférables dans les textes dits " homogènes ". Se pose la question de savoir s'il est possible pour le traducteur de rendre compte des représentations du texte source sans pour autant nuire à la communicabilité du texte cible. Bien plus, quelles stratégies s'offrent au traducteur de ce type de textes dans un contexte où la traduction peut parfois prendre des allures de "création parallèle " (Mopoho, 1997 : 258) ? De quelle marge de manœuvre dispose le traducteur face à cet exercice de création, dans la mesure où il n'existe pas toujours d'équivalent "vernaculaire " ou sociolectal dans la culture cible ? Et quand bien même une telle variété correspondante existerait, celle-ci ne jouirait pas forcément du même " prestige " et ne pourrait pas être utilisée à la même ampleur.

Dans le cadre de cette réflexion, nous formulons l'hypothèse principale suivante : le chiac présente certes des difficultés de traduction du fait de sa nature hybride et de sa personnalité linguistique en tant que parler vernaculaire ou sociolecte. Toutefois, ce code est traduisible, car il existe des moyens de résoudre les problèmes de traduction que ce genre de textes est susceptible de poser au traducteur. En d'autres termes, " parler d'intraduisibles n'implique nullement que les termes(1) en question [...] ne soient pas traduits et ne puissent pas l'être l'intraduisible, c'est plutôt ce qu'on ne cesse de (ne pas) traduire " Cassin (2004 : XVII).

\section{Petites difficultés d'existence et le chiac}

Le roman Petites difficultés d'existence (ci-après PDE), dont l'action se déroule dans la ville de Moncton, met en exergue les deux personnages principaux de l'œuvre, Carmen et Terry, qui se sont définitivement installés à Moncton après avoir séjourné en Europe. Devenus adultes et parents d'un enfant, Étienne, ces deux protagonistes se joignent à Zed, un ami du couple, ainsi qu'à d'autres personnes, dont Lionel Arsenault et Pomme, pour rénover une vieille "bâtisse " (Daigle, 2002 : 14) en vue d'en faire un centre culturel et des lofts. En réalité, PDE constitue le troisième opus d'une trilogie romanesque dans laquelle France Daigle a expressément recours au chiac, en particulier dans les passages dialogaux. Si dans Pas pire (1998) on note un emploi assez limité du chiac, et dans une certaine mesure dans Un fin passage (2001), c'est dans PDE que le recours au vernaculaire atteint des niveaux jamais égalés dans l'œuvre de Daigle, ce qui sans doute a amené Leclerc (2008 : 173) à affirmer qu'" on peut lire dans Petites difficultés d'existence l'aboutissement d'un processus qui vise à légitimer sinon le chiac lui-même, du moins son usage littéraire ".

En effet, la légitimation du chiac dans PDE se manifeste sous plusieurs formes. Pour étayer notre propos, nous nous appuyons sur deux exemples. Le personnage de Sylvia Arsenault,

(1) Nous préférons utiliser le mot " parlers " car dans la présente étude, nous nous intéressons à la traduction du chiac en tant que code linguistique et non en tant que "termes". 
qui doit se battre contre le sentiment d'insécurité linguistique qu'elle entretient en français, est littéralement séduite par l'aisance avec laquelle Ludmilla Zablonsky, une française qui a récemment emménagé à Moncton avec son époux, s'exprime en français " hexagonal ". La voix narrative rend compte des impressions de Sylvia en ces termes : "Comme elle s'exprimait bien! Sylvia avait l'impression d'entendre couler un ruisseau. Cela lui donnait envie de roucouler pareillement. " (Daigle : 51). La même voix narrative, qui est mise à contribution dans le travail de légitimation du chiac dans l'œuvre de Daigle, valorise la variété de français qui pourtant, aux yeux de Sylvia, est moins prestigieuse que le français de "référence " : "Sylvia avait beau faire attention, dire "est-ce que" au lieu de "c'est-ti", elle n'entendait pas la musique de son propre langage. " (Daigle, ibid.).

De plus, le travail de légitimation du vernaculaire chiac à l'œuvre dans le roman de France Daigle prend une autre dimension lorsqu'Étienne Zablonsky, l'époux de Ludmilla, donc un étranger, s'exprime également en chiac lorsqu'il s'adresse à Zed : " Jokes-tu ? (Daigle : 141) ; et la voix narrative d'ajouter : "Zed trouva qu'il s'acclimatait bien " (Daigle, ibid.). Pas étonnant que l'utilisation du chiac par Étienne Zablonsky se situe dans le chapitre intitulé « Humilité ". Effectivement, le fait qu'Étienne Zablonsky s'exprime également en chiac peut être interprété comme une forme d'humilité qui participe d'une stratégie d'acceptation de la diversité linguistique exprimée dans le roman par la présence d'autres variétés de français (chiac, régionalismes acadiens d'origine française) aux côtés du français " central ». On le voit, les personnages du roman et la voix narrative contribuent activement à la légitimation du chiac dans PDE. D'ailleurs, ces personnages s'accommodent bien du plurilinguisme, en particulier de l'anglais, pour obtenir des formes linguistiques originales en chiac. À ce sujet, Leclerc (2008: 179-180) écrit :

D'un côté, c'est désormais sans honte que l'influence de l'anglais est revendiquée. Cette influence est source de création; elle donne lieu à des jeux de mots poétiques, comme lorsque Pomme, un ami de Terry et Carmen qui s'intéresse à l'art explique que le peintre Van Gogh "a commencé à peinturer après avoir perdu sa job. " Pomme remarque : "en anglais y disont fired. Mis au feu. Ou allumé, c'est selon" (Daigle: 163).

De ce qui précède, il ressort que le chiac fait l'objet d'une entreprise de légitimation dans PDE. Cependant, ce travail de reconnaissance ne va pas sans paradoxes. D'abord, le chiac dans l'œuvre de Daigle est réservé aux scènes de dialogues, et la langue de la narration est assurée en français standard. En outre, sur le plan graphique, l'anglais qui constitue une des composantes linguistiques essentielles du chiac est systématiquement marqué par l'italique, comme dans les extraits ci-après:

"Pis le petit Étienne ? Y'est assez cute. " (Daigle : 26)

"Worry pas, ma belle ! Juste worry pas, tout' va right ben aller! Tu vas voir. " (Daigle : 64) 
En effet, ce phénomène de rupture graphique dans la chaîne écrite ne s'applique pas aux régionalismes auxquels le chiac emprunte souvent certaines formes linguistiques, comme dans les deux exemples ci-dessous où les mots " plusse " et "faise ", qui sont attestés en français acadien, ne font l'objet d'aucune forme de démarcation :

"Awh! Encore plusse cute..." (Daigle : 36)

"Faut même que je faise attention de pas trop brosser par le fond. " (Daigle : 36)

Le fait de marquer au moyen de formes comme l'italique des éléments anglais, et par ricochet ceux qui ressortissent au chiac, participe de ce que Authier-Revuz et al. (2003) qualifient de " connotation autonymique ", ou encore de "figures d'ajout " (2002), ce qui ouvre la voie à quelques possibles interprétatifs. En effet, pour paraphraser Tandia et Tsofack (2009) évoquant la situation du camfranglais, l'emploi du chiac aux moyens d'aspérités graphiques peut être interprété comme la volonté de Daigle d'ériger une frontière entre le discours "vrai ", mis en place par la " scène générique " des institutions littéraires dominantes qui incarnent l'idéologie du "français standard ", et le discours informel institué par la " scénographie des dialogues et confidences ", généré par les " autres ". Dans PDE par exemple, l'on assiste à une altercation entre Carmen et Terry au sujet de l'utilisation du chiac. En effet, la présence d'un enfant (Étienne) au sein du couple amène Carmen à poser le problème de l'utilisation du chiac à la maison. Elle déclare à cet effet : " Je pense à Étienne. C'est pas beau un enfant qui parle chiac. Un adulte c'est pas si pire. " (Daigle : 144). Les propos de Carmen tendent à faire du chiac une " parole sous surveillance " (Herschberg, 1993 : 103) et suggèrent par le même coup que leur fils Étienne devrait parler le français de "référence » incarné par les dictionnaires que Zed offrira plus tard au couple, tandis que le chiac peut être parlé par les " autres ", c'est-à-dire les adultes. Pour ce qui concerne Terry, l'abandon du chiac serait perçu à la fois comme une censure et une perte d'identité dans la mesure où il affirme : "Pis anyways, depuis quand c'est qu'y faut qu'on se force pour parler notre langue ? On ne peut-ti pas parler comme on veut ?" On le voit, le chiac se situe au centre d'une situation paradoxale, écartelé entre deux positions contradictoires : son acceptation-légitimation et son rejet-illégitimation. II peut donc être considéré comme " un parler autre venu d'ailleurs, de chez les autres et [qui] se bat encore pour sa reconnaissance - même si son usage est légitimé par une quête d'identité - du fait de son incursion dans un territoire linguistique autre " (Tandia et Tsofack, ibid. : 322). La situation d'ambivalence dans laquelle se situe le chiac pourrait s'appliquer aisément à celles des écrivains dominés en général, et au cas de Daigle en particulier. À ce sujet, Casanova (1999: 218) écrit :

Pour accéder à la reconnaissance littéraire, les écrivains dominés doivent donc se plier aux normes décrétées universellement par ceux-là même qui ont le monopole de l'universel. S'ils veulent être perçus, il leur faut produire et exhiber une différence, mais ne pas montrer ni revendiquer une distance trop grande qui les rendrait, elle aussi, imperceptibles. N'être ni trop près ni trop loin. Tous les écrivains linguistiquement dominés par la France ont fait cette expérience. 
En tant que traducteur, et donc co-auteur, comment Robert Majzels, le traducteur de PDE, parvient-il à gérer cette ambivalence en traduction?

\section{Life's Little Difficulties ou la traduction-légitimation}

Dano LeBlanc (2009), l'un des farouches défenseurs et promoteurs du chiac, exprimait dans son blog son souhait d'assister un jour à la création de ce qu'il a nommé "le Reverse chiac ", une espèce de chiac de langue anglaise, tout en se montrant sceptique quant aux chances d'un tel "rêve " de devenir réalité. En effet, expliquait-il,

J'wonder défois si il n'y a pas tchèque place, pro'bly une p'tite place dans l'monde ayousque les gens sont juste comme nous autres icitte dans le sud-est. Quand j'dis comme nous autres j'parle about la way qu'on parle... non, pas mal but juste comme nous autres quand-même, except reversé. En autres mots, une place ayousque la majorité de la population est francophone pis la minorité est anglaise. En plusse de d'ça, ils auraient été discriminaté against à cause de la way qu'ils s'expriment. Humm ... ouèlle, il y a une chance infinitésimale que le phénomène que je vais appeler le Reverse chiac, pourrait arriver. So, juste pour être clair comme sous sous-terre, il y aurait une structure de phrase anglaise et les verbes seraient des mots français accordés (itou) (non paginé).

Les passages dialogaux dans la traduction anglaise de PDE pourraient se lire comme une sorte de Reserve chiac qui se dévoile au fil des pages d'un roman où l'hétérogénéité occupe une place de choix, compensant ainsi l'absence d'un vernaculaire ou parler équivalent dans la culture cible : "C'est pas si bad, is it? " (p. 17) ; "Sleeping. I took him along to the café tantôt... I don't know if it's on account of the last storm, but le monde was grouchy là-bas. " (p. 115) ; "Ça fait que we didn't hang about for long " (p. 115). Le choix de Robert Majzels de recourir à un chiac expérimental, créé de toutes pièces, pourrait être interprété comme le souci du traducteur de rendre compte de l'hybridation linguistique caractéristique du chiac, et partant d'éviter de produire des traductions homogénéisantes qui non seulement masqueraient, mais nieraient par le même coup, l'hétérogénéité fonctionnelle du texte original. Bien plus, il s'agit pour le traducteur de restituer dans l'univers romanesque la réalité sociolinguistique du sud-est du Nouveau-Brunswick caractérisée par une situation diglossique marquée par la cohabitation du français et de l'anglais. En optant pour une stratégie de traduction basée sur une recréation du chiac, Majzels participe à sa manière à l'entreprise de construction de la langue littéraire acadienne qui, selon Masson (1997: 128), " n'est pas une langue établie ", mais également aux canons de cette langue en traduction anglaise. L'exercice d'innovation linguistique à l'œuvre dans la traduction anglaise de PDE n'est pas sans conséquences sur la graphie.

Nous avons indiqué plus haut le fait que dans le texte original, les formes linguistiques qui ressortissent à l'anglais, composante essentielle du chiac, sont marquées systématiquement 
par l'italique, un moyen qui traduit la situation paradoxale dans laquelle se trouve le chiac dans la mesure où la présence de ce parler dans le roman peut être interprétée comme une forme de légitimation. En même temps, le recours à l'italique vise à susciter à la lecture « une pesée critique sur le corps ainsi souligné " (Herschberg, 1993 : 103). Dans Life's Little Difficulties, force est de constater que Majzels met un terme au phénomène de rupture graphique employé par Daigle : "Zed got up cheerfully. "Bonne idée" (p. 18) ; "What is an intellectuel? Anyway? " (ibid.) ; "That would be up to chacun " (p. 23) ; "Would be nice to keep some of it, it's comme beau " (p. 24). En effet, si la présence de l'italique dans le texte original peut être considérée notamment comme le surgissement d'un corps étranger, la traduction de Majzels entend par contre rendre justice à la réalité sociolinguistique du sud-est du Nouveau-Brunswick marquée par le contact de langues où la porosité linguistique est une réalité indéniable. Car, comme le souligne fort opportunément Tessier (2001: 18),

[L]'anglais [...] s'y intègre [dans le texte français], non pas à la façon d'éléments exogènes destinés à faire couleur locale, [...] mais bien comme un outil d'écriture à part entière, avec ses découpages sémantiques, son réseau connotatif propre, et une palette sonore autre, permettant les combinaisons les plus audacieuses avec la langue d'accueil, le français.

On pourrait en dire de même de la présence remarquée du français dans Life's Little Difficulties. En effet, il ne s'agit pas pour Majzels d'» introduire des éléments étrangers dans la culture d'accueil ", comme le fait remarquer Leclerc (2008: 181) qui reprend Berman (1985) à son propre compte. Ainsi, l'élimination de toutes formes de ruptures graphiques doublée de la présence affichée du français dans la traduction de Majzels pourrait se lire comme le souci du traducteur d'être en phase avec le référent sociolinguistique acadien.

Par ailleurs, la cohabitation du français et de l'anglais, donc du chiac, dans l'univers romanesque de Life Little Difficulties peut également se justifier par la volonté de Majzels de prendre en compte dans sa traduction un public qui, à notre humble avis, a rarement retenu l'attention des théoriciens de la traduction en Acadie, notamment le public bilingue. En effet, la plupart des spécialistes du chiac ont toujours fait remarquer que le vernaculaire chiac véhicule essentiellement une identité francophone (Perrot 1998 ; Leclerc 2005 ; Boudreau 2009). Pourtant, Pöll (2009 : 194) déclare au sujet des locuteurs de ce parler :

Comme il s'agit de locuteurs bilingues, une partie de leur identité sociale est liée à la langue anglaise. Le chiac peut donc apparaître comme le véhicule d'une identité ethnologique liée non seulement au français mais aussi à l'anglais, langue omniprésente et incontournable dans la vie sociale.

On le voit, en accordant une place de choix à l'hétérogénéité du chiac dans la traduction de passages dialogaux, Robert Majzels fait le pari de cibler simultanément les publics anglophone et bilingue, ce dans un effort visant à légitimer davantage le chiac. Majzels semble avoir réussi cette conquête d'un public pluriel dans la mesure où à aucun moment dans le texte, la recréation du chiac et les stratégies de traduction qu'il propose ne portent préjudice à la 
communicabilité du texte cible. On pourrait alors se demander si une telle démarche de Majzels ne constitue pas les prémisses d'une nouvelle approche de la traduction du chiac.

\section{Vers une approche écologique de la traduction?}

Après avoir décrit avec force détails les différentes stratégies auxquelles ont recours Robert Majzels et Jo-Anne Elder, les principaux traducteurs de la littérature acadienne, plus exactement du chiac vers l'anglais, Leclerc (2007 [2005]) est parvenue à la conclusion faite par Simon (1997 : 194-195) quelques années auparavant, et selon laquelle, au Canada, les traductions d'œuvres issues du Canada français vers le Canada anglais ont généralement oscillé entre les approches ethnographiques et l'assimilation. Or la traduction de PDE semble indiquer clairement qu'en optant pour une stratégie de traduction fondée essentiellement sur l'hétérogénéité, Robert Majzels a voulu éviter de reproduire le schéma de la sempiternelle opposition binaire traduction sourciste/traduction cibliste. Majzels opte pour une troisième voie, celle du centre, basée sur l'hybridité, ce qui lui permet de produire des traductions qui ne sont ni entièrement ethnographiques ni entièrement homogénéisantes. D'où le recours à un chiac que le traducteur crée de toutes pièces, contrairement à l'écrivain du texte source dont le chiac s'efforce de coller à la réalité. En fait, les stratégies de traduction adoptées par Robert Majzels pourraient se déployer dans le cadre de ce que nous appelons une "approche écologique de la traduction ". En effet, "[t] out autant que l'écologie étudie le lien entre les éléments vivants dans un espace donné, l'écologie linguistique étudie le lien entre les langues, ou plutôt entre les personnes qui parlent certaines langues, à un endroit étudié " (Ehrhart : 2011). Une approche écologique de la traduction s'intéresserait donc au lien entre la diversité linguistique et la traduction destinée à un public pluriel, caractéristique des environnements marqués par le contact des langues. Les traductions produites dans de tels espaces nécessiteraient la prise en considération de l'altérité linguistique et culturelle des textes hybrides par l'intégration et le respect de l'Autre, à l'image de la diversité linguistique et culturelle de la ville de Moncton en général et de la région sud-est du Nouveau-Brunswick en particulier. La prise en compte de tels paramètres pourrait grandement contribuer à une gestion saine et équitable de l'altérité lors de la traduction de textes provenant d'espaces littéraires linguistiquement et culturellement dominés, comme dans le cas du roman étudié dans le présent article.

\section{En conclusion}

On peut dire, pour conclure, que le chiac est un code hybride parlé au sud-est du NouveauBrunswick, au Canada. Ce vernaculaire véhicule une identité non seulement francophone mais aussi bilingue. En dépit de l'hybridation linguistique qui le caractérise, il reste après tout tra- 
duisible, comme le démontre la traduction de Majzels qui, en fondant ses stratégies de traduction sur l'hétérogénéité, a su rendre compte de l'altérité et de la diversité linguistique et culturelle du texte source sans pour autant nuire à la communicabilité du texte cible. Il évite ainsi l'opposition binaire traduction homogénéisante/traduction assimilationniste en optant pour une voie intermédiaire, celle du centre.

henri.biahe@dal.ca

Titulaire d'un Master en traduction de l'École Supérieure de Traducteurs et Interprètes (ASTI) de Buea au Cameroun, Henri BIAHÉ prépare actuellement un Ph.D. en linguistique à Dalhousie University au Canada. Il a enseigné la sociolinguistique à Saint-Mary's University (Halifax, Canada) entre 2010 et 2011. Ses travaux portent sur la théorie de la traduction, le postcolonialisme et la traductologie, l'hétéroglossie littéraire et la sociolinguistique.

\section{Bibliographie}

AUTHIER-REVUZ Jacqueline, DOURY Marianne et REBOUL-TOURÉ Sandrine, 2003, Parler des mots. Le fait autonomyque en discours, Paris, Presses universitaires de la Sorbonne Nouvelle. AUTHIER-REVUZ et LALA Marie-Christine, 2002, (dirs.), Figures d'ajout : phrases, texte, écriture, Paris, Presses Universitaires de la Sorbonne Nouvelle.

BALL Jennifer, 2004, "Peter Pan meets A Clockwork Orange ". Ottawa Xpress, 30 septembre 2004. www.ottawaxpress.ca/stage.aspx?ilDArticle $=4334$

BARBARA Cassin, 2004, Vocabulaire des philosophies, éd. Seuil/Le Robert.

BERMAN Antoine, 1985, "La traduction comme épreuve de l'étranger ", Texte 4, pp. 68-81.

BOUDREAU Annette, 2008, "Le français parlé en Acadie : idéologies, représentations et pratiques ", in La langue française dans sa diversité, Gouvernement du Québec, pp. 59-73.

BOUDREAU Annette et GADET Françoise, 1998, "Attitudes en situations minoritaires. L'exemple de l'Acadie ", in QUEFFÉLEC Ambroise (dir.), Le français en Afrique, Recueil d'études offert en hommage à Suzane Lafage, Didier Érudition, $n^{\circ} 12$, pp. 55-56, www.unice.fr/LLF-CNRS/ofcaf/12/Boudreau.htm.

BOUDREAU Annette et BOUDREAU Raoul, 2004, "La littérature comme moyen de reconquête. L'exemple de l'Acadie ", Glottopol, n³, pp. 166-180.

CASANOVA Pascale, 1999, La république mondiale des lettres, Paris, Seuil.

DAIGLE France, 1998, Pas pire, Moncton, Éditions d'Acadie. 
DAIGLE France, 1999, Just Fine, tr, Robert Majzels, Toronto, Anansi.

DAIGLE France, 2001, Un fin passage, Montréal, Boréal.

DAIGLE France, 2002, A Fine Passage, tr. Robert Majzels, Toronto, Anansi.

DERRIDA Jacques, 1985, "Des Tours de Babel ", in GRAHAM Joseph F. (dir.), Difference in Translation, Ithaca (N.Y.) et Londres, Cornell University Press, pp. 209-248.

EHRHART Sabine, 2011, "L'écologie linguistique au Luxembourg ", in http://blog.sorosoro.org//\%e2\%80\%99ecologie-linguistique-au-luxembourg (non paginé). Consulté le 16 juin 2011.

HERSCHBERG-PIERROT Anne, 1993, Stylistique de la prose, Paris, Belin Sup.

LEBLANC Dano, 2009, "Le Reverse Chiac ", http://www.capacadie.com/blogues/acadieman/acadieman-diaries-le-reverse-chiac. Consulté le 6 octobre 2011.

LECLERC Catherine, 2008, "Le chiac, le Yi King, et l'entrecroisement des marges : Petites difficultés d'existence en traduction ", in MERKLE Denise, KOUSTAS Jane, NICHOLS Glen et SIMON Sherry (dir.), Traduire depuis les marges/Translating from the Margins, Québec, Nota bene, pp. 163-192.

LECLERC Catherine, 2007 [2005], "Between French and English, Between Ethnography and Assimilation: Strategies for Translating Moncton's Acadian Vernacular ", TTR, vol. XVIII, $n^{\circ} 2$, 2e semestre, pp. 161-192.

MALABORZA Sonya, 2006, "La Traduction du théâtre en Acadie - Parcours et tendances actuelles ", TTR : Traduction, Terminologie, Rédaction. 19.1, pp. 175-203.

MASSON Alain, 1997, "Une idée de la littérature acadienne ", in Revue de l'Université de Moncton, $\mathrm{n}^{\circ} 1$, vol. 30, pp. 125-132.

MOPOHO Raymond, 1997, "Vernacularisation et traduction des textes pragmatiques en Afrique ", Paul, St-Pierre (dir.). Langues, traduction et post-colonialisme. TTR. Vol. X, n 1, pp. 245-261. PARÉ François, 1992, Les littératures de l'exiguiité, Ottawa, Le Nordir.

PERROT Marie-Ève, 2001, „Bilinguisme en situation minoritaire et contact de langues : l'exemple du chiac ", Faits de langues, n 18, pp 129-137.

PERROT Marie-Ève, 2005, "Le chiac de Moncton : description synchronique et tendances évolutives " in VALDMAN A., AUGER J. et PISTON-HATLEN D. (dir.), Le français en Amérique du Nord : état présent, Québec, Presses de l'Université Laval, pp. 307-326. 
PÖLL Bernhard, 2009, " Formes d'hybridation et typologie des normes linguistiques : quel rapport ? Le chiac et le mitchif en comparaison ", in Normes et hybridation linguistiques en francophonie, Actes de la section 6 du Congrès de l'Association des francoromanistes allemands, Augsbourg 24-26 septembre 2008, PÖLL Bernhard/SCHAFROTH Elmar (eds.), Espaces discursifs, Paris, L'Harmattan, pp. 183-201.

SIMON Sherry, 1997, "Translation and cultural politics in Canada ", in RAMAKIRHSNA S. (dir.), Translation and Multilingualism: Post-Colonial Contexts, Delhi, Pencraft International, pp. 192204.

TANDIA Jean-Jacques et TSOFACK Benoît, 2009, "Langues, discours et paroles intergénérationnelles : le camfranglais dans un journal pour jeunes ", in ANDRIOT-SAILLANT C. (dir.), Paroles, langues et silences en héritage, Clermont-Ferrand, Presses universitaires Blaise Pascal, pp. 311-327.

TESSIER Jules, 2001, Américanité et francité : essais critiques sur les littératures d'expression française en Amérique du Nord, Ottawa, Le Nordir.

YOUNG Hilary, 2002, C'est either que tu parles francais, c'est either que tu parles anglais : A cognitive approach to Chiac as a contact language. Ph.D. dissertation, Rice University. 